\title{
Intraoperative use of tranexamic acid in the control of bleeding in long bone fracture surgeries: a meta-analysis
}

\author{
Manuel A. Gaspar Jr*, Marco Paolo G. Lasam \\ Department of Orthopaedics, Cagayan Valley Medical Center, Tuguegarao City, Cagayan, Philippines
}

Received: 25 January 2022

Accepted: 17 February 2022

*Correspondence:

Manuel A. Gaspar Jr,

E-mail: drmannygasparjr@gmail.com

Copyright: ( $)$ the author(s), publisher and licensee Medip Academy. This is an open-access article distributed under the terms of the Creative Commons Attribution Non-Commercial License, which permits unrestricted non-commercial use, distribution, and reproduction in any medium, provided the original work is properly cited.

\begin{abstract}
Tranexamic acid (TXA) has been used successfully in the practice of orthopedics to reduce perioperative blood loss, particularly in total hip and knee arthroplasty and spine surgery. The use of TXA has shown potential to reduce blood loss, transfusion rates and volumes, perioperative hemoglobin change and hospital-related costs at various degrees. This review aimed to evaluate the clinical results of the intraoperative use of TXA in long bone fracture surgeries. Relevant electronic articles were reviewed through a systematic search and selection process, resulting in the selection of five randomized control trials with a total of 295 participants. These studies compared the primary outcome of this metaanalysis, which was blood loss. Four of the studies included post-operative hemoglobin levels as their outcomes. Furthermore, two studies compared drop in hemoglobin levels, while three studies included blood transfusion events. Results showed a significant difference in terms of total blood loss when intraoperative administration of TXA was compared to placebo in long bone surgeries with a mean difference of $-76.94[-106.62,-47.26](95 \% \mathrm{CI})(\mathrm{p} \leq 0.00001)$. There was no significant difference in terms of post-operative hemoglobin levels $(\mathrm{p}=0.27)$ with a mean difference of $0.31[-0.24,0.86](95 \% \mathrm{CI})$. No significant difference was also shown between the two groups as to drop in hemoglobin levels $(\mathrm{p}=0.29)$ with a mean difference of $-0.34[-0.97,0.29](95 \% \mathrm{CI})$. This meta-analysis showed that intraoperative use of TXA given during long bone fracture surgeries can effectively reduce total blood loss and reduce risk of transfusion events or avoid transfusion events.
\end{abstract}

Keywords: Intraoperative use of tranexamic acid, Long bone fracture surgery, Blood loss, Control of bleeding, Metaanalysis

\section{INTRODUCTION}

TXA has been widely available for over 40 years, but only recently was it started to be used routinely in many surgical disciplines. ${ }^{3}$ It serves as a pharmacologic agent that acts through an antifibrinolytic mechanism to stabilize formed clots and reduce active bleeding. It has been used successfully in orthopedics to reduce perioperative blood loss, particularly in total hip and knee arthroplasty and spine surgery. ${ }^{10,11}$ Numerous research studies have reported favorable safety and efficacy for its use in orthopedic surgeries, however, there is still no universal standard on its administration and its use has not yet become the standard of practice. ${ }^{7}$ Nonetheless, literature has reported that complication rates on its use are low, specifically for both arterial and venous thromboembolic events, particularly deep venous thrombosis and pulmonary embolism. However, there remains a dearth of data on patients with higher-risk conditions. ${ }^{9,12-15}$

The use of TXA has shown potential to reduce blood loss, transfusion rates and volumes, perioperative hemoglobin change and hospital-related costs. ${ }^{4,8-11}$ In our local setting, any method that can increase the conservation of blood products, reduce patients' laboratory costs and shorten 
their hospital stays is going to be a major factor driving the cost savings for both patient and hospital.

This review aimed to evaluate the clinical results of the intraoperative use of TXA in long bone fracture surgeries. Specifically, it aimed to compare the amount of total blood loss after intraoperative administration of TXA and associate other clinical parameters including postoperative hemoglobin levels, drop in hemoglobin levels, and transfusion events; and determine whether there was heterogeneity in the results of studies in the use of TXA in the control of blood loss in long bone fracture surgeries.

\section{METHODS}

The following set of criteria was used in selecting articles: target population, adult individuals with open fracture reduction and fixation of the long bones (radius, ulna, humerus, femur or tibia); intervention, intraoperative use of TXA; primary outcome measure, total blood loss; the secondary outcomes included post-operative hemoglobin levels, drop in hemoglobin levels and transfusion events; and methodologic criteria, randomized controlled trial.

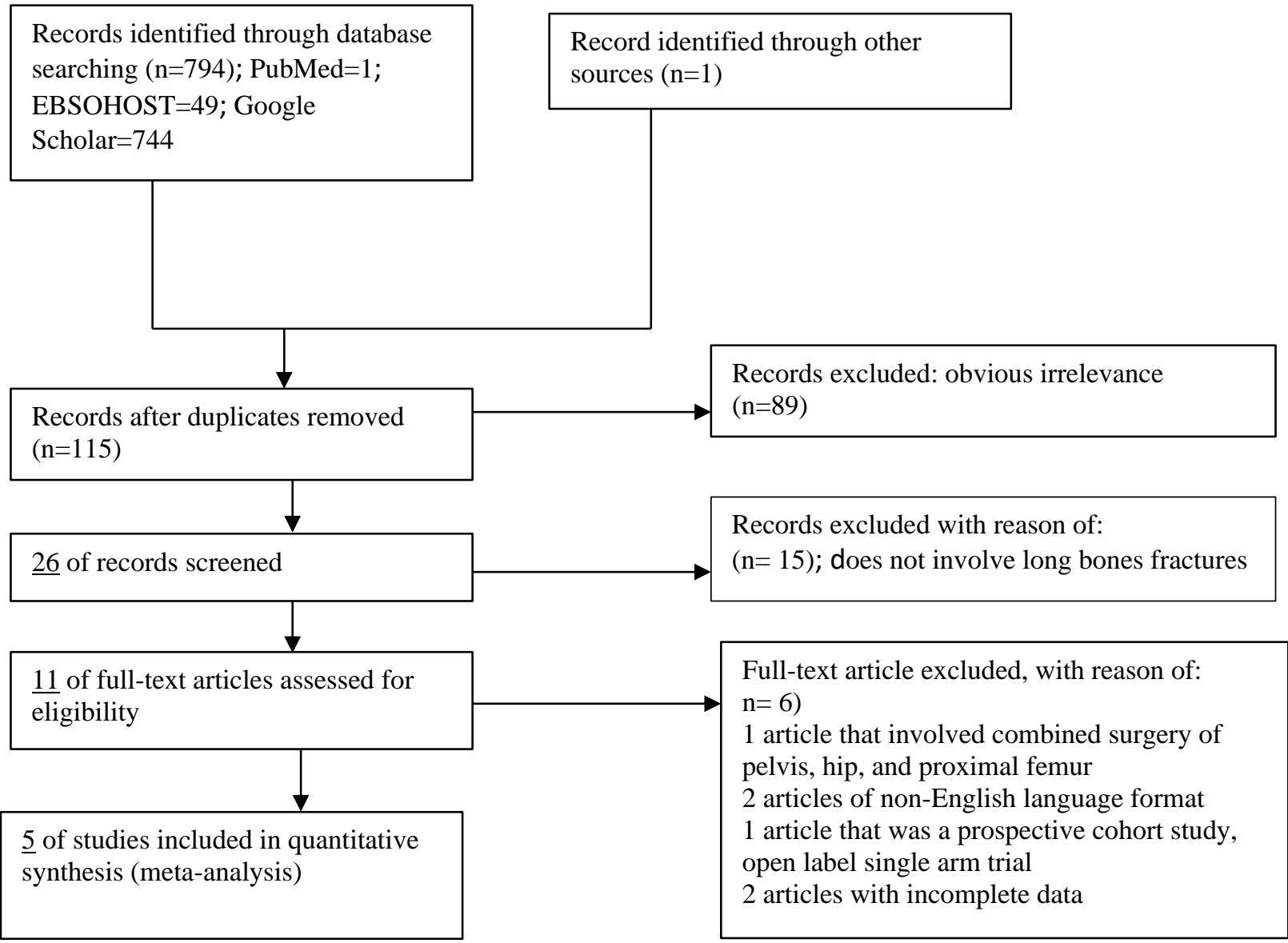

Figure 1: Process flow for the selection of potentially relevant studies.

The reviewers searched PubMed, Google Scholar, Cochrane database and EBSCO host for articles published from January 2015 to August 2020 using the set of criteria as search terms or keywords. Based on their titles, the articles were evaluated on whether or not they met the proposed study eligibility criteria. If the title suggested that the study might be relevant, the abstract was retrieved. All selected abstracts from the search were reviewed independently and potentially eligible studies were chosen for retrieval (Figure 1).

The reference lists of all key articles were also reviewed for additional papers, which were then retrieved and evaluated for eligibility. Additional strategies to supplement the article search included: manual searches of tables of contents of four major orthopaedic journals Journal of Bone and Joint Surgery (American and British), Journal of Orthopaedic Trauma and Clinical Orthopaedics and related research; bibliography review of two major trauma textbooks in orthopaedics (Rockwood and Green, fractures in adults; Browner, Jupiter and Swiontkowski, skeletal trauma); title review of presentations and posters in programs of three major orthopaedic meetings (American Academy of Orthopaedic Surgery, Orthopaedic Trauma Association, and Canadian Orthopaedic Association); and interviews with content experts 
(orthopaedic consultants of the institution). This process of judging the eligibility of articles was repeated for all potentially relevant studies.

The search strategy for the identification of studies was patterned after similar searches in the Cochrane Database whenever possible. The following subject-specific search strategy was combined with a strategy for identifying controlled trials and was modified for use in other databases: fracture/open reduction/or/1-2/long bone/femoral shaft/radial shaft/ulnar shaft/tibial shaft/humeral shaft and/3,4 and/3,5 and/3,6 and/3,7 and/3,8 and/3,9/intraoperative use of TXA/control of bleeding/blood loss or/16-18 and/10,19 and/11,19 and/12,19 and/13,19 and/14,19 and/15,19.

Articles were excluded for any of the following: randomized controlled trials which did not include the intraoperative use of TXA; abstracts for which the full text articles cannot be extracted; randomized controlled trials which did not include open fracture reduction and fixation of the long bones on their study population; articles that were not written in English; and articles with incomplete data.

\section{Methodological quality assessment}

The methodological quality for each study was independently assessed using the generic evaluation tool developed by the Cochrane musculoskeletal injuries group. Disagreements among the reviewers were resolved by consensus.

A 10-item score covering important aspects of internal and external validity was used. The items are listed below.

\section{Was the assigned treatment adequately concealed prior to allocation?}

$2=$ method did not allow disclosure of assignment; $1=$ small but possible chance of disclosure of assignment or unclear; $0=$ quasi-randomised or open list/tables; Cochrane code: clearly yes $=\mathrm{A}$; not sure $=\mathrm{B}$; clearly no $=\mathrm{C}$.

\section{Were the outcomes of patients/participants who withdrew described and included in the analysis (intention to treat)?}

$2=$ withdrawals well described and accounted for in the analysis; 1=withdrawals described and analysis not possible; $0=$ no mention, inadequate mention, or obvious differences and no adjustment.

\section{Were the outcome assessors blinded to treatment status?}

$2=$ effective action taken to blind assessors; $1=$ small or moderate chance of unblinding of assessors; $0=$ not mentioned or not possible.
Were the treatment and control group comparable at entry?

$2=$ good comparability of groups, or confounding adjusted for in analysis; $1=$ confounding small; mentioned but not adjusted for; $0=$ large potential for confounding, or not discussed.

Were care programmes, other than the trial options, identical?

$2=$ care programmes clearly identical; $1=$ clear but trivial differences; $0=$ not mentioned or clear and important differences in care programs.

Were the inclusion and exclusion criteria clearly defined?

$2=$ clearly defined; $1=$ inadequately defined; $0=$ not defined.

Were the interventions clearly defined?

$2=$ clearly defined interventions are applied with a standardised protocol; $1=$ clearly defined interventions are applied but the application protocol was not standardized; $0=$ intervention and/or application protocol are poorly or not defined

Were the outcome measures used clearly defined? (by outcome)

$2=$ clearly defined; $1=$ inadequately defined; $0=$ not defined.

Were diagnostic tests used in outcome assessment clinically useful? (by outcome)

2=optimal; 1 =adequate; $0=$ not defined, not adequate.

Was the surveillance active, and of clinically appropriate duration?

$2=$ active surveillance and appropriate duration; $1=$ active surveillance, but inadequate duration; $0=$ surveillance not active or not defined.

The results of the quality assessment were for qualitative guidance only and were not used to weigh any particular study in the analysis.

\section{Data extraction}

The full list of titles and abstracts identified by the electronic search was examined and information regarding the population, intervention and outcomes were extracted from each relevant article; full text reports of any study thought possibly relevant by any reviewer were obtained. In addition to the primary outcome as defined in the eligibility criteria, the reduction of hemoglobin concentration, clinical outcomes, blood coagulation 
values, thromboembolic complications and transfusion rates were also noted. The reviewers were not blinded to authorship or institution. Authors of included trials were contacted for further information if important data were not included in the trial report or if the full text was not available.

\section{Data analysis}

Data analysis using the REVMAN 5 software, which was the Cochrane collaboration's program for preparing and maintaining reviews was used. It allowed entry of protocols as well as full text reviews and can perform meta-analysis on the data entered. Data across studies were pooled and relative risks were calculated, associating $95 \%$ confidence intervals for each outcome using the random effects model of DerSimonian and Laird. This model assumed that the studies included in the review represent a random sample of all the potentially available studies at present and in the future.

\section{Evaluation of heterogeneity}

Major differences in the apparent effect size found across studies define important heterogeneity of study results. If important heterogeneity was found, the study populations, treatments, measures of outcome and methodology must be examined to determine the source. Heterogeneity may be due to differences in the populations (intraoperative use or non-use of TXA or the type of long bone affected) or differences in methodological features (methodological quality scores, completeness of follow up or whether studies were published or unpublished). For each potential determinant of heterogeneity, relative risks were compared across categories.

\section{RESULTS}

A total of 795 citations were found, 1 from PubMed, 744 from Google Scholar, 49 from EBSCOhost and 1 unpublished article. For femoral shaft fractures, the reviewed articles came from PubMed (1), EBSCOhost (24) and Google Scholar (295). For radial shaft, ulnar shaft and tibial shaft fractures, 136, 56 and 171 related literatures were retrieved solely from Google Scholar, respectively. For humeral shaft fractures, 25 articles from EBSCO host and 86 articles from Google Scholar were reviewed. From all study titles, 11 proved to be potentially eligible and were subjected to abstract review.

The search and selection process resulted in the inclusion of randomized controlled trials from five published full reports with a total of 295 participants Bantolo et al 2019; Haghighi et al 2017, Lei et al 2017, Mukherjee et al 2016 and Luo et al 2019. The reviewers attempted to contact the authors of the trials but were unsuccessful.

In three of the articles, patients were randomized into two groups: one group received TXA while the other group received placebo. Bantolo et al 2019 investigated 31 patients who were treated for an open reduction internal fixation with interlocked intramedullary nailing of the femur. Haghighi et al 2017 compared 40 patients who underwent femoral fracture operation and Lei et al 2017 investigated 77 patients who were treated with proximal femoral nail. Mukherjee et al 2016 compared the efficacy of TXA in reducing blood loss during orthopedic femoral surgeries among 59 patients. Luo et al 2019 compared the efficacy and safety of TXA in controlling bleeding during the surgical treatment of intertrochanteric fragility fracture with proximal femoral nail anti-rotation in 90 patients.

For the outcomes of each specific study, Haghighi et al 2017 had all four outcome measures: blood loss, required blood transfusion, hemoglobin level and drop in hemoglobin level while Bantolo et al 2019 had three: blood loss, drop in hematocrit level and drop in hemoglobin level. Lei et al 2017 only included blood loss, hemoglobin level, hematocrit level and blood transfusion, if the patient required one, as outcomes in his study while Mukherjee et al 2016 measured only blood loss and hemoglobin level. Lastly, Luo et al 2019 had three outcome measures included, namely blood loss, hemoglobin level and blood transfusion, if the patient required one.

The studies were difficult to compare mainly due to the variety of outcomes under investigation in each. Nevertheless, an overall comparison can be devised between the use and non-use of TXA in reducing blood loss in long bone fracture surgeries. Particularly, the five studies compared the primary outcome of this study, which was blood loss. Four studies, Haghighi et al 2017, Lei et al 2017, Mukherjee et al 2016 and Luo et al 2019 included post-operative hemoglobin levels as their outcomes. Furthermore, two studies, Bantolo et al 2019, Haghighi et al 2017 compared drop in hemoglobin levels while three studies, Haghighi et al 2017, Lei et al 2017 and Luo et al 2019 included whether or not the patient required blood transfusion.

Detailed information on total blood loss was available in all five published studies $(n=295)$. Pooled statistical analysis indicated a significant decrease in total blood loss with the intraoperative use of TXA as shown by the mean difference of $-76.94[-106.62,-47.26](95 \% \mathrm{CI})$ with a pvalue of $<0.00001$ compared to placebo (Table 2 ).

Information on secondary outcomes was available in all five studies as well. Despite the variation in the outcomes, pooling of results across studies was still possible with three secondary outcomes noted to be common: postoperative hemoglobin levels, drop in hemoglobin levels, and transfusion events.

For post-operative hemoglobin level, pooled statistical analysis showed no effect in the four studies (Haghighi et al 2017, Lei et al 2017, Mukherjee et al 2016 and Luo et al 2019 where this outcome was seen. The mean difference between the two groups (TXA versus placebo) was 0.31 [$0.24,0.86](95 \% \mathrm{CI})$ with a p value of 0.27 (Table 3 ). 
Similarly, for drop in hemoglobin levels, pooled results showed no significant effect of TXA use, with a mean difference value of -0.34 [-0.97,0.29] (95\% CI) (Table 4). This result was in contrast with the findings of Bantolo (2019) of a higher value in the placebo arm, which showed the favorable use of intraoperative TXA to decrease the drop in hemoglobin levels, with a mean difference value of $-0.59[-1.18,-0.00](95 \% \mathrm{CI})$ between the TXA group and the placebo group.

Table 1: Characteristics of included studies.

\begin{tabular}{|llllll|}
\hline Authors & TA & $\mathbf{P}$ & Design & Intervention & Outcomes \\
\hline Bantolo et al 2019 & 17 & 14 & RCT & TXAG/TXANG & BL, drop in Hct, drop in Hgb \\
\hline Haghighi et al 2017 & 18 & 20 & RCT & TXAG/TXANG & BL, RBT, CI, SS, Hgb, drop in Hgb, MAP, IHR \\
\hline Lei et al 2017 & 37 & 40 & RCT & TXAG/TXANG & BL, Hgb, Hct, RBT \\
\hline Mukherjee et al 2016 & 29 & 30 & RCT & TXAG/TXANG & BL, Hgb, BP, PHR \\
\hline Luo et al 2019 & 44 & 46 & RCT & TXAG/TXANG & BL, Hgb, RBT, HS \\
\hline Total participants & 145 & 150 & & & \\
\hline
\end{tabular}

TA-tranexamic acid given group; P-placebo; RCT-randomized controlled trial; TXAG-tranexamic acid given; TXANGtranexamic acid not given; BL-blood loss; Hct-hematocrit level; Hgb-hemoglobin level; RBT-required blood transfusion; CII-crystalloid infusion; SS-surgeon satisfaction; MAP-mean arterial pressure; IHR-intraoperative heart rate; BP-blood pressure; PHR-pre-operative and post-operative heart rate; HS-hospital stay.

Table 2: Pooled statistical analysis for total blood loss.

\begin{tabular}{|c|c|c|c|c|c|c|}
\hline \multirow{3}{*}{ Journals } & \multicolumn{4}{|c|}{ Total blood loss } & \multicolumn{2}{|c|}{ Total subjects } \\
\hline & \multicolumn{2}{|l|}{ TA } & \multicolumn{2}{|l|}{$\mathbf{P}$} & \multirow{2}{*}{ TA } & \multirow{2}{*}{$\mathbf{P}$} \\
\hline & Mean & SD & Mean & SD & & \\
\hline Bantolo et al 2019 & 394.12 & 42.87 & 478.57 & 108.69 & 17 & 14 \\
\hline Haghighi et al 2017 & 830.22 & 476.22 & 850.55 & 460.75 & 18 & 20 \\
\hline Lei et al 2017 & 98.30 & 59.10 & 128.85 & 123.18 & 37 & 40 \\
\hline Mukherjee et al 2016 & 323.28 & 45.28 & 411.67 & 41.38 & 29 & 30 \\
\hline Luo et al 2019 & 384.50 & 366.30 & 566.20 & 361.50 & 44 & 46 \\
\hline
\end{tabular}

Table 3: Pooled statistical analysis for post-operative hemoglobin levels.

\begin{tabular}{|c|c|c|c|c|c|c|}
\hline \multirow{3}{*}{ Journals } & \multicolumn{4}{|c|}{ Hemoglobin levels } & \multicolumn{2}{|c|}{ Total subjects } \\
\hline & \multicolumn{2}{|l|}{ TA } & \multicolumn{2}{|l|}{$\mathbf{P}$} & \multirow{2}{*}{ TA } & \multirow{2}{*}{$\mathbf{P}$} \\
\hline & Mean & SD & Mean & SD & & \\
\hline Haghighi et al 2017 & 10.27 & 1.90 & 10.37 & 1.58 & 18 & 20 \\
\hline Lei et al 2017 & 10.270 & 1.318 & 11.527 & 6.093 & 37 & 40 \\
\hline Mukherjee et al 2016 & 10.61 & 1.56 & 10.0721 & 1.62 & 29 & 30 \\
\hline Luo et al 2019 & 9.5 & 1.6 & 8.9 & 1.4 & 44 & 46 \\
\hline Total & & & & & 128 & 116 \\
\hline
\end{tabular}

Table 4: Pooled statistical analysis for drop in hemoglobin levels.

\begin{tabular}{|c|c|c|c|c|c|c|}
\hline \multirow{3}{*}{ Journals } & \multicolumn{4}{|c|}{ Drop in hemoglobin levels } & \multicolumn{2}{|c|}{ Total subjects } \\
\hline & \multicolumn{2}{|l|}{ TA } & \multicolumn{2}{|l|}{$\mathbf{P}$} & \multirow{2}{*}{ TA } & \multirow{2}{*}{$\mathbf{P}$} \\
\hline & Mean & SD & Mean & SD & & \\
\hline Bantolo et al 2019 & 1.42 & 0.63 & 2.01 & 0.97 & 17 & 14 \\
\hline Haghighi et al 2017 & 2.04 & 1.75 & 1.97 & 0.74 & 18 & 20 \\
\hline Total & & & & & 35 & 34 \\
\hline
\end{tabular}

Table 5: Pooled statistical analysis for transfusion events.

\begin{tabular}{|c|c|c|c|c|}
\hline \multirow{2}{*}{ Journals } & \multicolumn{2}{|c|}{ Transfusion events } & \multicolumn{2}{|c|}{ Total subjects } \\
\hline & TA & $\mathbf{P}$ & TA & $\mathbf{P}$ \\
\hline Haghighi et al 2017 & 1 & 6 & 18 & 20 \\
\hline Lei et al 2017 & 10 & 22 & 37 & 40 \\
\hline Luo et al 2019 & 7 & 17 & 44 & 46 \\
\hline Total & 18 & 45 & 99 & 106 \\
\hline
\end{tabular}


For transfusion events, pooled statistical analysis showed that the risk ratio for transfusion events for the three studies was $0.45[0.28,0.71](95 \% \mathrm{CI})$. The use of intraoperative TXA did not incur increased risks for transfusion events compared to placebo. A relative risk reduction of 0.55 (1-RR) indicates that the use of intraoperative TXA will reduce the risk of patients of having transfusion events by $55 \%$ as compared to placebo. This risk difference translates into an NNT of 4, which means that for every four patients given TXA intraoperatively for the control of blood loss, one transfusion event could be avoided. Furthermore, a p-value of 0.0006 indicates that there was a significant difference between the treatment and placebo groups as to transfusion events.

\section{DISCUSSION}

To date, this study was the first attempt on meta-analysis of randomized controlled trials on the intraoperative use of TXA in the control of blood loss in long bone fracture surgeries. Other meta-analysis reviewed the effect of TXA mostly on procedures such as total knee arthroplasty and total hip arthroplasty. TXA was a pharmacologic agent that acted through an antifibrinolytic mechanism to stabilize formed clots and reduced active bleeding. Intravenous TXA reduced blood loss in patients with surgical bleeding, thereby reducing the need for transfusion.

As stated by Mukherjee et al 2016 prevention of blood loss with the use of antifibrinolytic agents had been proven in several studies in different types of surgeries except for commonly performed orthopedic surgeries like those involving long bone fractures. He further stated that these agents were effective in the reduction of blood loss and the requirement of perioperative transfusion of blood products. This was also concurred by other studies in this meta-analysis. ${ }^{5-9}$ Accounts of thrombotic events were found in three randomized controlled trials but these are of no significant difference when compared to placebo, hence no increased risks were reported. ${ }^{6,7,9}$

Based on the statistical analyses made in this paper, there was significant evidence that the intraoperative administration of TXA compared to placebo results in lesser total blood loss in long bone surgeries. This was explained by TXA accumulating in the extracellular space, thus, inhibiting tissue fibrinolysis and accordingly stabilizing the clot and more importantly, having no effect on coagulation parameters. ${ }^{6}$ A plasma concentration of TXA was required to suppress fibrinolysis. ${ }^{8}$ All the trials included in this meta-analysis used intravenous administration of TXA probably achieving plasma concentrations and efficiently suppressing fibrinolysis, thereby decreasing the total blood loss.

The non-significant differences in terms of post-operative hemoglobin levels and the drop in hemoglobin levels when comparing TXA and placebo did not support any deleterious effect of its use in long bone surgeries. However, it was noteworthy that the use of intraoperative TXA had beneficial effects in terms of reducing the risk for transfusion events among patients who underwent long bone surgeries by $55 \%$ as compared to placebo use.

Although this may be the largest pooled data from recent prospective trials of which we were aware, the number of patients in each group may not be large enough to demonstrated differences of small magnitude. On the basis of the results in this study, it seemed likely that any relative advantage associated with either TXA use versus placebo was small and may only emerge in a study with much larger numbers of patients.

\section{CONCLUSION}

This review described different outcomes across various eligible studies which showed that there id a significant difference in terms of total blood loss when TXA is compared with placebo. There is also some evidence that shows that the use of intraoperative TXA reduces the risk for transfusion events. The journals reviewed showed insufficient number of participants with the same outcome measure to be able to suggest for a universal standard on the administration of TXA and make it a standard of practice for long bone surgeries.

\section{Recommendations}

It is recommended that the date for the significant outcome measures in the studies in future trials in this field be reported in such a way that further analyses and comparisons can be made. Moreover, comparative studies regarding the intraoperative use of varying dosages of TXA in the control of bleeding in long bone fracture surgeries can also be undertaken. It is also recommended that more randomized controlled trials on the intraoperative use of TXA be pursued so a stronger conclusion may be achieved, and that the results of this meta-analysis may be used as reference.

\section{ACKNOWLEDGEMENTS}

The Authors wish to acknowledge the Contributions of Dr. Rei Ubiña and Mr. Airon Calica in the accomplishment of this study. To my dearly departed father, Dr. Manuel G. Gaspar, Sr., CESE - for your invaluable insights on this research. May you be equally proud of our last engagement together.

\section{Funding: No funding sources \\ Conflict of interest: None declared \\ Ethical approval: Not required}

\section{REFERENCES}

1. $\mathrm{Ng} \mathrm{W}$, Jerath A, Wąsowicz M. Tranexamic acid: a clinical review. Anaesthesiol Intensive Ther. 2015;47(4):339-50. 
2. Hunt BJ. The current place of tranexamic acid in the management of bleeding. Anaesthesia. 2015;70(1):50-8.

3. Robb PJ. Tranexamic acid-a useful drug in ENT surgery? J Laryngol Otol. 2014;128(7):574-9.

4. Lin ZX, Woolf SK. Safety, efficacy, and costeffectiveness of tranexamic acid in orthopedic surgery. Orthopedics. 2016;39(2):119-30.

5. Bantolo A, Detoyato LYK (2019). Tranexamic acid pre-operative prophylaxis to reduce bleeding during open reduction internal fixation with interlocked intramedullary nailing of the femur: a randomized control study. Int J Scientif Res Publicat. 2019;10(10):102-9.

6. Haghighi M, Ettehad H, Mardani-Kivi M, Mirbolook A, Nabi BN, Moghaddam R, et al. Does tranexamic acid reduce bleeding during femoral fracture operation? Arch Bone Joint Surg. 2017;5(2):103-8.

7. Lei J, Zhang B, Cong Y, Zhuang Y, Wei X, Fu Y, et al. Tranexamic acid reduces hidden blood loss in the treatment of intertrochanteric fractures with PFNA: a single-center randomized controlled trial. J Orthop Surg Res. 2017;12(1):124.

8. Mukherjee $\mathrm{M}$, Biswas $\mathrm{C}$, Chatterjee $\mathrm{S}$, Bandyopadhyay BK. Comparative study of efficacy of reduction of blood loss by tranexamic acid and epsilon aminocaproic acid for orthopedic femoral surgeries. Anaesth Pain Intensive Care. 2016;20(4):417-21.

9. Luo X, He S, Lin Z, Li Z, Huang C, Li Q. Efficacy and safety of tranexamic acid for controlling bleeding during surgical treatment of intertrochanteric fragility fracture with proximal femoral nail antirotation: a randomized controlled trial. Indian J Orthopaed. 2019;53(2):263-9.

10. Delanois R, Mont M. (2013) Does tranexamic acid reduce blood loss in total knee arthroplasty? commentary on an article by X. Aguilera, MD, et al.: efficacy and safety of fibrin glue and tranexamic acid to prevent postoperative blood loss in total knee arthroplasty. a randomized controlled clinical trial. J Bone Joint Surg. 2013;95(22):179.

11. Eva Y.W. Tse, MBChB etal.(2011) Current Concepts Review Reducing Perioperative Blood Loss and Allogeneic Blood Transfusion in Patients Undergoing Major Spine Surgery Department of Anaesthesiology and the Department of Orthopaedics and Traumatology, The University of Hong Kong, Hong Kong SAR, China THE JOURNAL OF BONE AND JOINT SURGERY, INCORPORATED

12. Tse EYW, Cheung WY, Ng KFJ, Luk KDK. Oral and intravenous tranexamic acid are equivalent at reducing blood loss following total hip arthroplasty a randomized controlled trial Rush university medical center, Chicago, Illinois. J Bone Joint Surg Am. 2011;93(13):1268-77.

13. Gill JB, Chin Y, Levin A, Feng D. The use of antifibrinolytic agents in spine surgery a metaanalysis. J Bone Joint Surg Am. 2008;90(11):2399407.

14. Napolitano LM, Cohen MJ, Cotton BA, Schreiber MA, Moore EE. Tranexamic acid in trauma: how should we use it? J Trauma Acute Care Surg. 2013;74(6):1575-86.

15. Ponnusamy KE, Kim TJ, Khanuja HS. Current concepts review perioperative blood transfusions in orthopaedic surgery. J Bone Joint Surg Am. 2014;96(21):1836-44.

Cite this article as: Gaspar Jr MA, Lasam MPG. Intraoperative use of tranexamic acid in the control of bleeding in long bone fracture surgeries: a metaanalysis. Int J Res Orthop 2022;8:233-9. 